\title{
Über den Sehpurpur im tapezierten Auge.*
}

\author{
Von \\ Yuji Hosoya. \\ (細 谷 雄 二) \\ (Aus dem II. plysiologischen Institut der Universität zu Sendai, \\ Direktor : Prof. Dr. T. Fujita).
}

\section{Einleitung.}

Schon in 1897 und 1898 wurde die Erkennbarkeit des Sehpurpurs bei Teleosteer (Abramis, Acerina u. a.) und Krokodil (Alligator), die Tapetum retinale besitzen, von $\mathrm{G}$. A belsd or $\mathrm{ff}^{\left.1{ }^{22}\right)}$ ophthalmoskopisch nachgewiesen. Beim Tapetum retinale ist ${ }^{3445)}$ das vor demselben gelegene Netzhautepithel mehr oder weniger stark pigmentiert. Aber beim Tapetum chorioideale ist das Epithel des Aussenblatts der Netzhaut, wie ich schon mitteilte, ${ }^{6)}$ in grossem Umfange des Augenhintergrundes völlig pigmentlos.§

Da das tapezierte Auge hinter der Netzhaut einen spiegelnden Reflektor hat, steht es also in ganz verschiedenem Verhältnis mit dem nichttapezierten, z. B. beim Menschen, bei welchem die Netzhaut mit schwarzem Chorioidealpigment belegt ist. Daher ist es sehr interessant, über das Verhalten des Sehpurpurs in dieser pigmentlosen Netzhautpartie zu erfahren, jedoch wurde darüber bis jetst noch nicht eingehender studiert. Um die Lücke in diesbezüglichen Untersuchungen ausfüllen zu vermögen, habe

*) Der wesentliche Inhalt mit Demonstration wurde im japanischen Physiologenkongress 1926 (J. of Biophysics, 1927, 2, cxvir) mitgeteilt.

1) G. Abelsdorff, Zeitschr. f. Psychol. u. Physiol. d. Sinnesorg., 1897, 14, 77.

2) Derselbe, Arch. f. (Anat. u.) Physiol., 1898, 155.

3) J. I. Chieritz, Arch. f. Anat. (u. Physiol.), 1889, Suppl. Bd., 164.

4) II. Laurens u. S. R. Detwiler, J. of Exp. Zool., 1921, 32, 213.

5) S. Garten, Die Verïnderungen der Netzhaut durch Licht. Grae fe-Sa em ischs Hb. d. ges. Augenheilk., 2. Autl. I. Teil, III. Bd. Kap. XII, Anhang. S. 108.

6) Y. Ho so y a, dies. J., 1929, 12, 131.

\$) Betreffs der Pigmentlosigkeit des tapezierten Gebietes ist zu bemerken, dass auch diese Netzhautteile beim Pferde nicht ganz pigmentfrei sind, sondern schwarze oder graue Pigmentkörner im ganzen Netzhautepithel, und zwar immer weniger dicht nach der Mitte des Gebiets, aufweisen, so dass man nur von einer Pigmentarmut sprechen darf. 
ich die folgenden Versuche mit den Säugetieren und den Selachier ansgeführt.

\section{Ophthalmoskopische Beobachtungen des Sehpurpurs am lebenden Auge.}

Ich versuchte den Farbenwechsel des Sehpurpurs in der Netzhant der lebenden Säugetiere, und zwar der Katze und des Hundes, ophthalmoskopisch nachzuweisen, was aber noch nicht gelang, da die Anhäufung des Sehpurpurs bei beiden nicht so intensiv ist, dass der Purpurton des Augenhintergrundes mittels des Ophthalmoskops deutlich in die Augen des Untersuchenden fällt. Für diese Untersuchung bietet das Selachierauge ein sehr geeignetes Material, indem die purpurne Fürbung der Net zhaut direkt oberhalb des Sehnerveneintritts, der bereits erwähnten pigmentlosen Strecke entsprechend, einen merkwürdig tief roten Streifen sog. Sehleiste bildet (vgl. Fig. 1).

Die Pupille des Cynias ist rundlich und verengert sieh beim Lichteinfall zum horizontalen Streifen. Das Auge des lebenden Tieres wurde mittels des Tageslichtes, das durch das Fenster des Dunkelzimmers auf den Versuchstisch kam, in folgender Weise ophthalmoskopiert. Nachdem der Fisch etwa 4 Stunden lang im Dunkeln verweilt hatte, wurde er in ein breites Tuch eingewickelt und in Versuchiswamne gut gefesselt. Bei der Einstellung des Ophthalmoskops zeigt das Dunkelauge einen schönen purpurnen Fundus, in welchem ein längs der Sehleiste gezogener purpurner Streifen auf den ersten Blick auffällt. Die purpurne Farbe der Netzhaut bleicht im Laufe des 20 minutigen Hellaufenthaltes des Fisches allmählich aus, und nähert sich der des Tapetums, so dass einige Nctzhautgefässe erkenubar werden, während das tiefe Rot der Sehleiste meist lange zurückbleibt. Wenn der Fisch nochmals ins Dunkelzimmer gebracht und ungefähr 2 Stunden lang stehen gelassen wird, so tritt der verlorene Purpur mit gleicher Deutlichkeit wie am Aufange wieder auf.

2. Beobachtungen des Sehpurpurs am enukleierten Bulbus und an der isolierten Retina.

Zu diesem Versuche wurden zahlreiche Augen von Säugetieren (Katzen, Hunde, Rinder und Pferde) und des Selachiers (Cynias und Raja) angewandt. Die Farbe der Dunkelretina ist beim Hunde und bei der Katze sehr schwach dunkelrot, bein Rinde tief rot, beim Pferde schwach rot und beim Selachier purpurn (vgl. Figg. 1 u. 2). 
Die Anhäufung des Sehpurpurs ist bei Säugetieren, abgesehen von Rindern, bei welchen sie besonders intensiv ist, wie oben erwähnt, relativ

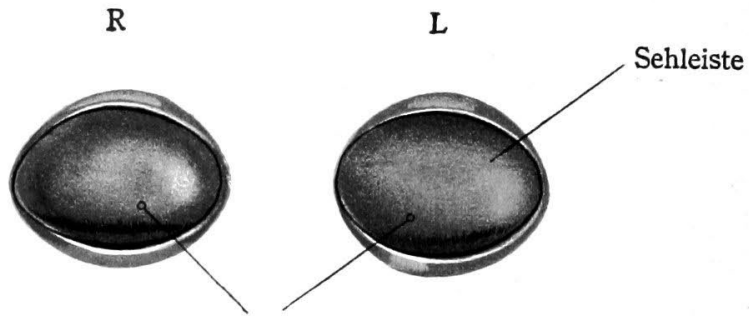

Papilla n. optici

Fig. 1.

Hintere Hälften der beiden Augen eines Cynias manazo Bleeker. R: Im IIellen enukleiert. L : Nach der Enukleation 60 Minuten lang im Dunkeln aufbewahrt.
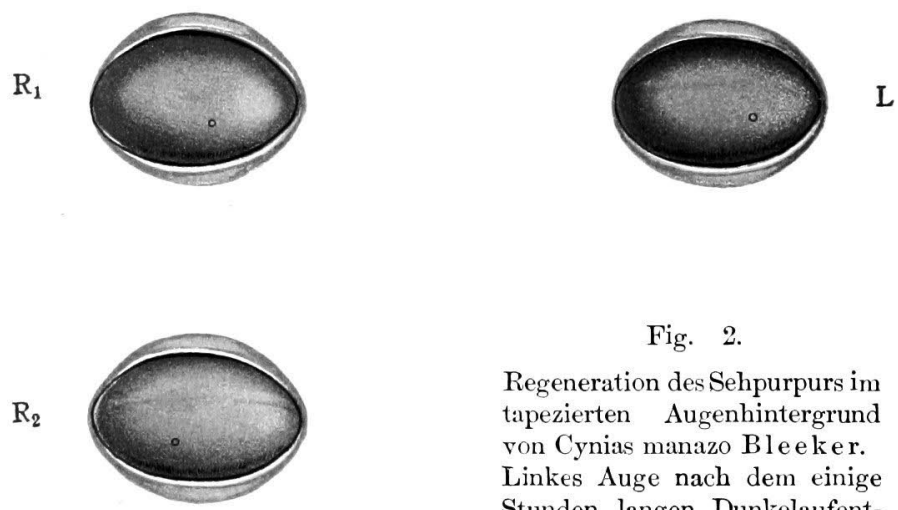

Fig. 2 .

Regeneration des Sehpurpurs im tapezierten Augenhintergrund von Cynias manazo Bleeker. Linkes Auge nach dem einige Stunden langen Dunkelaufenthalt des Fisches enukleiert. L : ungebleichte linke hintere Hïlfte. Das rechte Auge $\left(\mathbf{R}_{1}\right)$ wurde nach 30 Minuten langer Belichtung exstirpiert und gleich darauf im Dunkeln 1 Stunde lang $\left(R_{2}\right)$ und nachher noch 2 Stunden lang $\left(\mathbf{R}_{3}\right)$ aufbewahrt. 
schwach, und der Blutgehalt der Netzhant wirkt bei der Beobachtung desselben mehr oder woniger störend. So muss der Bulbus vor jedem Versuche möglichst blutfrei gemacht werden. Dazu werden die Versuchstiere (Süugetiere) direkt nach dem Tode von Halsgefüssen entblutet. Die Augen werden vor dem Tode etwa 30 Minuten lang mit der Augenbinde bedeckt. Sofort nach dem Schlachten oder Verblutungstode enukleierte ich die Augenbulbi, die dann in Ringerlösung aufbewahrt und im Dunkelzimmer bei rotem Lichte äquatorial halbiert wurden. Wenn die hintere Hälfte des Bulbus nach dem Ausstürzen des Glaskörpers in eine mit Ringerlösung gefüllte weisse Porzellanschale gebracht und unter dem mässig verdunkelten Tageslichte beobachtet wird, so zeigt sich, dass die Säugetiernetzhaut auch in tapezierten Gebiete, wo das Pigment in der Pigmentepithelschicht günzlich fehlt, noch den Sehpurpur enthält. Am Lichte bleicht die Farbe der Netzhaut relativ schnell aus, und an ihrer Stelle tritt die Irideszenz des darunter gelegenen Tapetums hervor, wodurch. die genanere Betrachtung der Netzhautfarbe gestört wird.

Ich habe an Süugeraugen auch daraufhin untersucht, ob etwa in der tapezierten, pigmentlosen Netzhautpartie die Sehpurpurausbleichung stärker resp. schneller ₹or sich gehe, als in der pigmentierten, was der Fall sein müste, wenn die Lichtwirkung in der ersteren durch geringere Absorption und st:irkere Reflexion stärker wïre, als bei der letzteren. Es war noch kein bestimmtes Resultat zu erzielen, da bei mehrere Minuten langer Belichtung mit Sonnen- oder Lampenlicht kein erkennbarer Farbenunterschied zwischen beiden zu stande kam.

Um die Ausbreitung des Sehpurpurs in der Netzhaut und seine Ausbleichung durch das Licht näher zu untersuchen, wurde das im Dunkeln gehaltene Rinderauge unmittelbar nach der Enukleation in 3-5.o Formol lichtdicht aufbewahrt und nach 24 Stunden im $\dddot{1}$ quator halbiert. Das Ablösen der Netzhaut von der Chorioidea ist dann leicht auszuführen, was sonst ohne Zerstörung der ersteren schwer möglich ist. Die abgelöste Net\%haut bleibt bein Herausstürzen des Glaskörpers an der Opticuspapilla anhaftend. Durch diese Fixation, wie A belsdor $\mathrm{f}^{8 /}$ bei Fischangen und $\mathrm{N}$. Andogs $\mathrm{y}^{93}$ bei Kaninchenaugen angegeben hat, wurde auch der Schpurpur der Rindernetzhaut so lichtbeständig, dass er annähernd 15 Minuten lang unter dem hellen Lichte fast unverïndert besteht, also ein zur Demonstration geeignetes Material darbietet. Nach etwa 20 minutiger Belichtung bleicht die purpurne Farbe der Netzhaut etwas schneller und binnen ca. 40 Minuten gänzlich aus. Dabei konnte ich nicmals das Torhanden-

7) X. Andogsky, Arch. f. Ophthalnol, 1897, 44, 422.

S) G. Abelsdorff, Zeitschr. f. Psychol. u. Physiol. d. Sinnesorg., 1897, 14, $8 \%$.

9) N. Andogsk y (7), 422 . 
sein eines gelblichen intermediären Stadiums, des sog. Sehgelbs, nachweisen.

Bei der Verbreitung des Sehpurpurs ist es besonders merkwürdig, dass die purpurne Färbung der Süugernetzhaut in einer schmalen Zone, der sog. Sehleiste, am intensivsten ist. Diese ist bei Kaninchen direkt unterhalb, bei sonstigen genannten Tieren aber oberhalb des Sehnerveneintritts horizontal angezogen.

\section{Regeneration des Sehpurpurs in der pigmentlosen Netzhautpartie.}

Beim Sïugretierauge (Rind, Pferd, Katze und Hund) ist die Regeneration des Selıpurpurs nach der Enukleation wie W. C. Areys und W. $K \ddot{~} \mathrm{~h} n \mathrm{e}^{10)}$ beim Kaninchen angegeben haben, schwer nachweisbar, während beim Selachierauge diese nicht nur an der halbierten hinteren Hälften des Bulbus, sondern auch an der isolierten Netzhant noch gut erkennbar ist.

Um die Zeitdauer des Dunkelaufenthaltes, während dessen der Sehpurpurgehalt das Maximum erreicht, zu prïfen, wurden mehrere Selachier im Dunkeln gelassen, und nach verschieden langem Zeitintervall konnte man die Netzhautfarbe des enukleierten Augenbulbus beobachten.

Wenn das 'Tier nach dem Verweilen im Hellen ungetähr eine Stunde lang lichtdicht in Seewasser blieb, so zeigte die Netzhaut des Tieres eine tiefrote (ein wenig zum Purpur neigende) Farbe, welche in dieser Zeit schon ihr Maximum erreicht zu haben scheint, denn auch bei längerem, mehrere Stunden dauerndem Dunkelaufenthalt nimmt die Farbentiefe nicht mehr zu. Freilich ist die Regeneration des Sehpurpurs vom Erbaltenbleiben des Kreislaufes abhängig und folglich tritt beim exstirpierten Hellauge, wie unten erörtert wird, die vollständige Regeneration erst nach 2 stündiger Dunkelaufbewahrung ein.

Um die Regenerationszeit eingehender zu untersuchen, enukleierte ich dem einige Stunden lang im Dunkeln schwimmenden Cynias oder Raja beide Augen und bewahrte das eine derselben sofort in einer mit Seewasser gefüllten Flasche im Dunkeln auf. Das andere habe ich zuerst im hellen Zimmer 30 Minuten lang dem diffusen Tageslichte ausgesetzt und unmittelbar darauf in der genannten Flasche lichtdicht aufbewahrt. In bestimmtem Zeitintervall nach der Herstellung einer Anzahl solcher enukleierten Augenpare $\left(10^{\prime}, 20^{\prime}, 30^{\prime}, 60^{\prime}, 120^{\prime}\right.$, etc. nach dem Einbringen des Hellauges ins Dunkel) halbierte ich je ein Paar der 2, 215.

10) W. C. Areys u. W. Kühne, Untersuch. a. d. physiol. Inst. Heidelberg, 1878, 
so präparierten Augen (d.h. Dunkel- und Hellbulbus desselben Tieres) äquatorial und verglich die Farbentöne des Hintergrundes miteinander. Innerhalb eines 30 minutigen Zeitraumes ist der Farbenunterschied zwischen dem Hell- und Dunkelauge ganz deutlich, und zwar zeigt das erstere blaugrünliche (durchschimmernde Tapetumfarbe) und das letztere schöne Purpurfarbe. Wenn das Zeitintervall 60 Minuten überschreitet, nimmt die Rötung der Hellretina inmer mehr zu, so dass interessanterweise solcher Unterschied nach etwa 2 stündiger Dunkelaufbewahrung vollkommen verloren geht und die Farbe des Tapetumgebietes beider Augen gleich tief purpurn wird (vgl. Fig. 2).

Andererseits ist aber der Sehpurpur der Selachier schwerer ausbleichbar. Auch wenn der Fisch meherere Stunden lang bei guter Tagesbeleuchtung im Zimmer belassen wird, zeigt die isolierte Netzhaut noch mehr oder weniger deutlich eine rötliche Färbung, und das herausgenommene Auge muss besonders stark beleuchtet werden, um den Sehpurpur spurlos auszubleichen.

Auch die isolierte Retina des Selachiers ist noch regenerationsfähig, allerdings muss sie zur Wiedererhaltung des tiefen Purpurs über 5 Stunden lang im Dunkeln gehalten werden. Wenn ich auch nachweisen konnte, dass das Netzhautepithel an der Tapetumfliche der Chorioidea, wovon die Netzhaut entfernt war, anhaftend blieb, war ich dennoch nicht sicher, ob die letztere ganz epithelfrei war oder nicht. Jedenfalls fand die Regeneration des Sehpurpurs derselben immer überall gleichmüssig statt. Die schwach rote Netzhautfarbe des im Hellen verweilten Selachierauges hat keinen Stich ins Violett, sondern ist mehr zum gelblichroten Ton geneigt, und solange dieser noch vorhanden ist, erfolgt, wie Ayres und $K$ ühne ${ }^{11)}$ beim Kaninchenauge angegeben haben, die vollkommene Erholung des Purpurs viel schneller als nach vollstiindiger Ausbleichung.

Aus den vorgehenden Befunden ist ohne weiteres zu folgern, dass die Regeneration des Sehpurpurs sich auch in der tapezierten Partie der Netzhaut, wo das Pigment in der Pigmentepithelschicht fehlt, vollzieht. Die Tatsache, dass auch bei Säugetieren der pigmentfreie Netzhautteil Sehpurpur enthält, spricht für die von W. Trendelenburg, ${ }^{12}$ sowie $\mathrm{H}$. Laurens und $\mathrm{S}$. R. Detwiler ${ }^{13}$ bei anderen Tierarten ausgesprochenen Ansichten, und die, dass gerade diesem Netzhautteil wohl die grösste Sehfunktion zukommt, spricht, wie S. Garten ${ }^{14)}$ hervorgehoben hat, gegen

11) W. C. A yres u. W. Kühne (10), 294 .

12) W. Trendelenburg, Ergeb. d. Physiol, 1911, 11, 19.

13) H. Laurens u. S. R. Detwiler (4), 221.

14) S. Garten, Zeitschr. f. Augenheilk., 1922, 47, 187. 
die Ansicht, dass die Lichtenergie zuerst vom Netzhautpigment absorbiert wird und dann ihre Reizwirkung auf die Sehzellen entfaltet (z. B. Schanzsche $e^{15}$ Theorie).

\section{Zusammenfassung.}

(1) Der Sehpurpurgehalt der Netzhaut ist am lebeuden tapezierten Selachierauge, bei dem scine Anhïufung sehr intensir ist, ophthalmoskopisch nachweisbar.

(2) Die Netzhant des im mïssig hellen Tageslichte lebenden Tieranges (besonders dentlich beim Selachier) zeigt mehr oller weniger purpurne Nuance.

(3) Auch im Tapetumgebiet, wo das Pigment in der Netzhautepithelschicht völlig fehlt, enthalten die Sïugetier- und Selachierretina Sehpurpur, weleher ohne Termittlung des Pigments gut regenerierbar ist.

(4) Der Sehpurpur des lebenden Selachiers regeneriert vollkommen in 1 stündigem Dunkelaufenthalt, der des enukleierten Auges in 2 und der der isolierten (epithelfreien?) Retina erst nach 5 Stunden Dunkelaufbewahrung.

15) F.Schanz, Münch. med. Wuchensehr, 1921, 1890, Zeitschr. f. Augenheilk., 1921, 46, 811, Zeitschr. f. Sinnesphysiol, 1983, 54, 98 und Zeitschr. f. Physik, 1923, 12, 28. 